\title{
A comparison of thresholds for $1 / 3$-octave filtered clicks and noise bursts in infants and adults
}

\author{
KATHLEEN M. BERG \\ University of Florida, Gainesville, Florida
}

\begin{abstract}
Behavioral thresholds of 6-month-old infants and adults were determined for $1 / 3$-octave filtered clicks and 300-msec noise bursts with center frequencies ranging from .5 to $8 \mathrm{kHz}$. For noise bursts, differences between infant and adult thresholds were largest at low frequencies and smallest at $8 \mathrm{kHz}$. For clicks, infants' thresholds were most like adults' at $4 \mathrm{kHz}$, and age differences in creased at both lower and higher frequencies. Differences between click and noise thresholds were significantly larger for infants than for adults at .5, 1 , and $8 \mathrm{kHz}$, but not at 2 and $4 \mathrm{kHz}$. These results suggest that improvements in threshold for long-duration stimuli during infancy may not be accompanied by comparable changes in threshold at short durations. The delayed development of sensitivity to low- and high-frequency clicks appears consistent with maturational trends recently described for the auditory brainstem response.
\end{abstract}

Within recent years, numerous studies of auditory development have established that age-related improvements in sensitivity do not occur uniformly across frequency. For a wide variety of mammalian and avian species, sensitivity is initially greatest for stimuli in the low- to midfrequency range and improves at both lower and higher frequencies with further development (Rubel, Born, Deitch, \& Durham, 1984). In human infants, this sequence is characterized by a rapid increase in sensitivity to high-frequency stimuli during the first months of life, followed by a much more gradual improvement in thresholds for low frequencies (Olsho, Koch, Carter, Halpin, \& Spetner, 1988; Schneider, Trehub, \& Bull, 1980; Trehub, Schneider, \& Endman, 1980; Werner \& Feeney, 1990; Werner \& Gillenwater, 1990). The shape of the audibility curve, the function relating absolute sensitivity to stimulus frequency, appears adult-like by 6 months of age (Olsho et al., 1988), although improvement in sensitivity continues well into childhood, with high-frequency performance approaching, and perhaps exceeding, adult levels by 4-5 years and low-frequency thresholds continuing to decrease until approximately 10 years of age (Elliott \& Katz, 1980; Schneider, Trehub, Morrongiello, \& Thorpe, 1986; Trehub, Schneider, Morrongiello, \& Thorpe, 1988).

For the most part, studies of auditory sensitivity during infancy and early childhood have employed relatively long-lasting stimuli, in excess of $100-200 \mathrm{msec}$ in duration. However, work recently reported by Gray (1990) suggests that maturation of sensitivity to short- and long-

This work was supported, in part, by a grant from the March of Dimes Foundation and by NIH Grant R01-DC00017. Thanks are due Randy Limer, Susan Pastina, Tracy Marsh, and Kathy Shoucair for their assistance in testing subjects. Correspondence may be sent to the author at the Institute for Advanced Study of the Communication Processes, 63 Dauer Hall, University of Florida, Gainesville, FL 32611. duration stimuli may not occur simultaneously. In a study of auditory development in young chicks, Gray found agerelated reductions in threshold for stimuli longer than approximately $250 \mathrm{msec}$ in duration, whereas thresholds for briefer stimuli remained unchanged. To account for these findings, he proposed that auditory sensitivity matures in stages, improving at long durations first and at short durations sometime later.

At present, the few available data on development of sensitivity to brief stimuli in human infants come primarily from studies of the auditory brainstem response (ABR) to brief clicks. The ABR consists of a series of lowamplitude potentials generated by activation of the auditory nerve and structures of the auditory brainstem during the first $10 \mathrm{msec}$ following delivery of an auditory stimulus (Moore, 1987). It shows little variation over a wide range of stimulus durations and is therefore considered to be solely an onset response (Hecox, Squires, \& Galambos, 1976). Several investigators have reported delayed maturation of the ABR to high-frequency clicks in infants and young children (see, e.g., Folsom \& Wynne, 1986, 1987; Teas, Klein, \& Kramer, 1982), and the recent work of Eggermont and associates (Ponton, Eggermont, Coupland, \& Winkelaar, 1992) indicates that maturation of responses to low-frequency clicks may be delayed as well. Although these results suggest that development of both the ABR and behavioral measures of sensitivity are characterized by similar frequency-specific trends, the relationship between the two remains uncertain. The present experiment addressed this question by examining infants' behavioral thresholds for brief clicks similar to those employed in studies of the brainstem response. One goal was to determine if the pattern of delayed maturation at low and high frequencies described by Ponton et al. (1992) for the ABR would also be evident in behavioral thresholds for clicks in young infants. A second objective was to compare the audibility curves for brief clicks 
and longer duration stimuli in infants and adults. If, as Gray (1990) suggests, maturation of auditory sensitivity proceeds in duration-specific stages, then infants' audibility curves for short- and long-duration stimuli may differ in shape, reflecting the delayed development of sensitivity to stimuli that are very brief. The experimental paradigm was a visually reinforced operant procedure. Stimuli were $1 / 3$-octave filtered clicks and noise bursts with center frequencies ranging from .5 to $8 \mathrm{kHz}$.

\section{METHOD}

\section{Subjects}

A total of 69 normal, healthy infants 26-32 weeks of age served as subjects. They were randomly assigned to one of five frequency groups and received both the filtered-click and noise-burst conditions in counterbalanced order. Within a session, testing was continued either until acceptable threshold estimates had been obtained for both conditions or until the infant became agitated or disinterested as determined by preset criteria. Parents of infants successfully tested on only a single condition during their initial visit to the laboratory were asked to return for a second session. Forty infants ( 8 per group) completed the procedure successfully, requiring a mean of 1.2 sessions. This final sample consisted of 21 males and 19 females (mean age $=28.0$ weeks) whose distribution by age and sex across the five frequency groups was approximately equal. Of the 29 infants excluded from the final sample, 1 was replaced because of equipment failure, 3 failed to meet the initial criteria for conditioning described below, and 25 lost interest in the visual reinforcers before testing could be completed.

Forty young adult subjects ( 16 males, 24 females) were tested under the same conditions for comparison. They were enrolled in introductory psychology courses and received class credit for their participation.

\section{Apparatus and Procedure}

Test stimuli were 1 -octave filtered clicks and 300 -msec noise bursts with center frequencies of $.5,1,2,4$, and $8 \mathrm{kHz}$. Click stimuli were generated by "ringing" an analog filter ( $24 \mathrm{~dB} /$ octave rolloff) set to pass a $1 / 3$-octave band. The frequency spectra of filtered-click stimuli have been previously described by a number of investigators (e.g., Coats, Martin, \& Kidder, 1979; Folsom, 1984; Zerlin \& Naunton, 1975). Briefly, the spectra of 13 -octave filtered clicks, like those of longer duration filtered noise bursts, are determined by the characteristics of the filter: spectral amplitude is maximal at center frequency, $3 \mathrm{~dB}$ down at the nominal cutoff frequencies, and decreases at a rate determined by the rolloff of the filter for frequencies above and below the $\$$-octave passband. Like the spectra of filtered noise bursts, click spectra become broader with increasing center frequency as the width of $1 / 3$-octave bands increases. However, since the frequency and time-domain responses of a filter are related as a Fourier transform pair, the duration of filtered clicks varies inversely with bandwidth and thus necessarily decreases as center frequency increases. For a limited range of values, variations in pulse duration at the input of a filter affect the amplitude. but not the duration of stimuli at the output (Zerlin \& Naunton, 1975). In the present study, the durations of single, positive-going dc pulses were adjusted to produce symmetrical waveforms of maximum amplitude. These were .5. $4, .2, .1$, and . $1 \mathrm{msec}$ for center frequencies from .5 to $8 \mathrm{kHz}$, respectively. At the output of the filter, all waveforms consisted of approximately 4 cycles of the stimulus frequency: 1 at plateau and 3 within rising and falling portions of the envelope. Click intensity levels were calibrated in terms of the sound pressure level of a same-frequency continuous tone with equivalent peak-to-peak amplinude and are therefore expressed in decibels "peak-equivalent" sound pressure level (dB peSPL).
For waveforms symmetrical about the zero-pressure level, peakequivalent SPL is $3 \mathrm{~dB}$ lower than peak SPL, the intensity at the peak of the pressure change (Stapells, Picton, \& Smith, 1982).

Filtered noise bursts were produced by passing broadband noise through the same analog filter used to generate clicks. The bandwidths of noise bursts, like those of filtered clicks, increased in proportion to center frequency. They were gated with a rise/decay time of $3 \mathrm{msec}$. Both clicks and noise bursts were delivered in trains of seven per trial. Onset-to-onset interval of individual stimuli was $600 \mathrm{msec}$, resulting in a train approximately $4 \mathrm{sec}$ in duration. All stimuli were presented in sound field via an Advent loudspeaker (Model 5002). Stimulus level, measured at the site of the infant's head by a Quest 215 sound-level meter, did not vary more than $\pm 2 \mathrm{~dB}$ within the range of possible head positions.

Each infant was tested while seated on his/her parent's lap in the center of an IAC sound-attenuating chamber. The loudspeaker and three battery-operated toys in Plexiglas boxes were located $90^{\circ}$ to the infant's left, in a corner of the room adjacent to a one-way window. One experimenter, seated facing the infant across a small table, manipulated colorful toys to engage the infant's attention at midline and initiated trials when state and head position were judged appropriate. A second experimenter seated outside the chamber viewed the infant through the one-way glass and made judgments on head turns. During testing, both the parent and experimenter inside the test booth received masking noise through headphones to eliminate possible sources of bias.

Each trial consisted of a 4-sec observation interval, during which the stimulus train was presented with a probability of .5 . Head turns to the left during the observation interval were judged as positive responses, and failures to turn were considered negative responses. Head turns on signal trials were immediately reinforced by the 3-sec illumination of colored lights and activation of the toy within one of the Plexiglas boxes. The order of signal and nonsignal trials was random, with the restriction that no more than three trials of either type be presented consecutively. As a result of this restriction on trial sequences, information regarding trial type was available to adult experimenters on a small percentage of trials. Although specific feedback was not provided, experimenters were able to infer hits and false alarms by noting consequences of the infant's behavior. Probe trials (described below) introduced some additional uncertainty into trial sequences. However, trial type was predictable immediately following either three false alarms or four hits on consecutive trials. These combinations of events occurred very infrequently.

Prior to threshold estimation, each infant was required to meet a conditioning criterion with signal level fixed at $60 \mathrm{~dB}$ SPL for 300-msec noise bursts or $80 \mathrm{~dB}$ peSPL for clicks. This criterion was set at five consecutive correct responses for the initial stimulus condition and at two consecutive correct responses for the second condition. Once the criterion had been met, thresholds were determined using an adaptive one up-two down tracking procedure. Signal level was initially set $20 \mathrm{~dB}$ above adult threshold for the same condition and varied in 8-dB steps until the first up-down reversal. Step size was then reduced to $4 \mathrm{~dB}$, and tracking continued, with two consecutive correct responses required for a 4-dB decrease in level and a single failure to respond resulting in a 4-dB increase in level. Outcomes on nonsignal trials did not contribute to alterations of signal level. Intervals between trials were varied to ensure that the temporal sequence of signal and nonsignal trials was not predictable, and trials were initiated only when the infant was judged to be in the appropriate state, best described as "mildly interested" in the objects displayed at midline. If the infant became obviously distracted during the observation interval, the trial was repeated.

After the first reversal, suprathreshold probe trials with the signal set at conditioning level were presented every sixth trial to assess the infant's interest in the visual reinforcer. Tracking continued until the fourth up-down reversal, and threshold estimates were computed as the mean stimulus level over the last two reversals. If false alarms occurred on more than one third of the nonsignal 
trials, or if the infant failed to respond on more than two probe trials, the threshold estimate was considered unacceptable and the stimulus condition was repeated. Average length of sessions was approximately $20 \mathrm{~min}$.

Adult subjects were provided with a response box and instructed to press a button whenever they heard the stimulus train. Intertrial intervals varied randomly from 1 to $15 \mathrm{sec}$ in duration so that time of signal presentation was uncertain. Feedback for correct detections was provided by the brief activation of lights within one of the Plexiglas boxes. All other procedures were identical to those described for infants.

\section{RESULTS}

Threshold estimates for $1 / 3$-octave filtered clicks and noise bursts are shown for each of the five frequency groups in Figure 1. ${ }^{1}$ Error bars represent \pm 1 standard deviation from the mean. In general, adult thresholds for both click and noise stimuli were in good agreement with results previously described in the literature. Filtered noise thresholds were 3-4 $\mathrm{dB}$ higher than ISO standards for tones presented in free field (Killion, 1978), but nearly identical to free-field thresholds reported by Robinson and Whittle (1964) for octave bands of noise. The audibility curve for filtered clicks was roughly parallel to that for noise bursts, in agreement with the results of both Yost and Klein (1979) and Zerlin and Naunton (1975). Although differences in procedure and measurement conventions across laboratories make specific comparisons difficult, click thresholds were well within the range of values reported by other investigators for stimuli having similar characteristics (see, e.g., Folsom \& Wynne, 1987; Stapells et al., 1982).

As expected on the basis of previous work, infants' thresholds were higher than those of adult subjects, especially for filtered clicks. ${ }^{2}$ Audibility curves for click stimuli were clearly consistent with the frequency-specific pattern of maturational delay described for the ABR. Infants' click thresholds were most like adults' at $4 \mathrm{kHz}$,

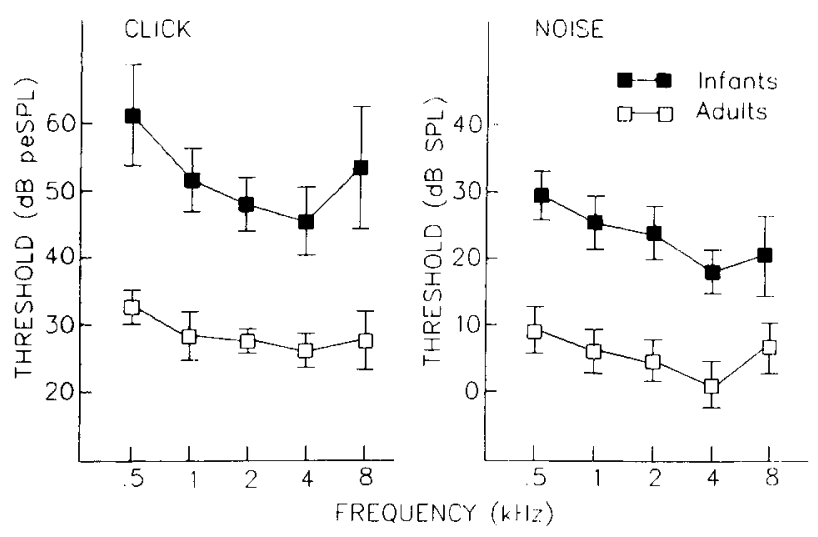

Figure 1. Infant and adult thresholds for $1 / 3$-octave filtered clicks and noise bursts with center frequencies ranging from .5 to $8 \mathrm{kHz}$. Noise thresholds are in $\mathrm{dB}$ SPL; click thresholds are in $\mathrm{dB}$ peSPL. Error bars represent \pm 1 standard deviation from the mean. and age differences increased at both lower and higher frequencies; adult-infant threshold differences were 28.5, 19.5, and $26 \mathrm{~dB}$ for .5-, 4-, and 8-kHz clicks, respectively. In contrast, differences between infant and adult thresholds for noise bursts were largest at low frequencies and smallest at high frequencies; mean infant threshold was approximately $20 \mathrm{~dB}$ above adult threshold for $.5-\mathrm{kHz}$ noise bursts, but only $14 \mathrm{~dB}$ above adult threshold for $8-\mathrm{kHz}$ stimuli. An analysis of variance (age $\times$ frequency $\times$ stimulus type) confirmed that these differences in the shape of audibility curves for clicks and noise bursts were significant $[F(4,70)=3.36, p<.025]$. When results obtained at each frequency were analyzed separately, click-noise threshold differences were found to be significantly larger for infants than for adults at $.5 \mathrm{kHz}$ $[F(1,14)=11.23, p<.005], 1 \mathrm{kHz}[F(1,14)=4.78$, $p<.05]$, and $8 \mathrm{kHz}[F(1,14)=14.66, p<.005]$, but not at 2 and $4 \mathrm{kHz}$.

Audibility curves for clicks and bursts of noise were also reliably different for infant subjects alone [frequency $X$ stimulus type, $F(4,35)=2.80, p<.05$ ]. Specific comparisons indicated that differences between infants' thresholds for clicks and noise bursts were significantly larger at both $.5 \mathrm{kHz}[F(1,14)=6.84, p<.025]$ and $8 \mathrm{kHz}[F(1,14)=6.29, p<.05]$ than at $2 \mathrm{kHz}$.

There were no reliable differences in incidence of false alarms across either frequency or duration conditions, although overall false-alarm rate was significantly higher for infants $(M=.18)$ than for adults $(M=.02)[F(1,70)=$ $94.54, p<.001] .^{3}$

\section{DISCUSSION}

When infants' thresholds for filtered clicks are compared with those of adult subjects, it is clear that behavioral thresholds for brief clicks exhibit the same frequency-specific developmental trends described by Ponton et al. (1992) for the ABR; infants' click thresholds are most adult-like for stimuli in the mid-frequency range and less mature at both lower and higher frequencies. Furthermore, a comparison of click and noise thresholds indicates that infants' sensitivity to low- and high-frequency clicks is also less mature than their sensitivity to $300-\mathrm{msec}$ noise bursts with the same center frequency. It is unlikely that this pattern of differences between click and noise thresholds is due to frequency-specific differences in the duration of filtered clicks, for two reasons. First, since click duration increased as center frequency decreased, clicknoise threshold differences would be expected to become smaller rather than larger at lower frequencies; second, there is no evidence of a comparable difference in audibility curves for clicks and noise bursts in adult subjects.

Werner and associates have recently reported a series of studies on the development of thresholds for pure-tone stimuli during infancy. Their results indicate that very young infants 2 to 5 weeks of age are more sensitive to .5- and $1-\mathrm{kHz}$ tones than to $4-\mathrm{kHz}$ stimuli (Werner \& Feeney, 1990; Werner \& Gillenwater, 1990). Between 
1 and 3 months of age, there is a substantial increase in sensitivity to $4-\mathrm{kHz}$ tones (Olsho et al., 1988; Werner \& Gillenwater, 1990), and the period between 3 and 6 months is characterized by a marked improvement in threshold at $8 \mathrm{kHz}$ (Olsho et al., 1988). Audibility curves for filtered noise bursts in the present study are very similar to the pure-tone audibility curves reported by Olsho et al. for subjects of comparable age. In both instances, thresholds of 6-month-old infants are most adult-like at $8 \mathrm{kHz}$ and most elevated at low frequencies. Infants' click thresholds, on the other hand, are significantly elevated at both .5 and $8 \mathrm{kHz}$ and define an audibility curve much more like that reported by Olsho et al. for 3-month-olds. The delay in maturation of sensitivity to brief low- and high-frequency clicks appears consistent with Gray's (1990) multistage model of auditory development and suggests that in human infants, just as in young chicks, improvements in threshold for long-duration stimuli may not be accompanied by comparable changes in threshold at short durations.

In their recent extensive investigation of the $A B R$ in infants and young children, Ponton et al. (1992) used a highpass-noise masking technique to obtain derived responses from specific regions along the basilar membrane and examined developmental changes in the Wave IWave $\mathrm{V}$ interpeak interval. They found that age-related changes in this measure of brainstem conduction time progressed more slowly and reached maturity later for very low and high frequency clicks than for stimuli in the mid-frequency range. According to their estimates, midfrequency channels of the brainstem reach maturity by 12-15 months, whereas low- and high-frequency channels are not functionally mature until approximately 2 years of age. Both the extended time course of these changes and the symmetrical low-high pattern of maturational delay are consistent with click thresholds obtained in the present study and suggest the possibility that development of behavioral thresholds for clicks may parallel maturation of conduction time in brainstem pathways. In adult subjects, discrepancies between ABR and audiometric thresholds are generally attributed to the transient nature of the $A B R$ and its dependence upon the synchrony of neural discharge at stimulus onset (see, e.g., Gorga, Worthington, Reiland, Beauchaine, \& Goldgar, 1985; Worthington \& Peters, 1980). It may be that immaturities in neural synchrony also contribute to the delay in development of behavioral thresholds when stimuli are very brief.

\section{REFERENCES}

BERG, K. M. (1991). Auditory temporal summation in infants and adults: Effects of stimulus bandwidth and masking noise. Perception \& Psychophysics, 50, 314-320.

Conts, A., Martin, J., \& Kidder, H. (1979). Normal short-latency electrophysiological filtered click responses recorded from vertex and external auditory meatus. Joumal of the Acoustical Society of America. $65,747-758$.

Elliott, L., Katz, D. (1980). Children's pure-tone detection. Journal of the Acoustical Society of America, 67, 343-344.

Folsom, R. (1984). Frequency specificity of human auditory brainstem responses as revealed by pure-tone masking profiles. Journal of the Acoustical Society of America, 75, 919-924.

Folsom, R., \& WNNE, M. (1986). Auditory brain stem responses from human adults and infants: Restriction of frequency contribution by notched-noise masking. Journal of the Acoustical Society of America, 80, 1057-1064.

Folsom, R., \&YNNE, M. (1987). Auditory brain stem responses from human adults and infants: Wave $\mathrm{V}$ tuning curves. Journal of the Acoustical Society of America, 81, 412-417.

Gorga, M., Worthington, D., Reiland, J., Beauchaine, K., \& GOLDGAR, D. (1985). Some comparisons between auditory brain stem response thresholds, latencies, and the pure-tone audiogram. Ear \& Hearing, 6, 105-112.

Gray, L. (1990). Development of temporal integration in newborn chickens. Hearing Research, 45, 169-178.

Hecox, K., SQuires, N., \& Galambos, R. (1976). Brainstem auditory evoked responses in man: I. Effect of stimulus rise-fall time and duration. Journal of the Acoustical Society of America, 60, 1187-1192.

KILLION, M. (1978). Revised estimate of minimum audible pressure: Where is the "missing $6 \mathrm{~dB}$ "? Journal of the Acoustical Society of America, 63, 1501-1508.

MoORE, J. K. (1987). The human auditory brain stem as a generator of auditory evoked potentials. Hearing Research, 29, 33-43.

Olsho, L., Koch, E., Carter, E., Halpin, C., \& Spetner, N. (1988). Pure-tone sensitivity of human infants. Joumal of the Acoustical Society of America, 84, 1316-1324.

Ponton, C., Eggermont, J., Coupland, S., \& Winkelaar, R. (1992). Frequency-specific maturation of the eighth nerve and brainstem auditory pathway: Evidence from derived auditory brain-stem responses (ABR). Journal of the Acoustical Society of America, 91, 1576-1586.

RoBinson, D., \& Whittle, L. (1964). The loudness of octave bands of noise. Acustica, 14, 24-35.

Rubel, E., Born, D., Deitch, J., \& Durham, D. (1984). Recent advances toward understanding auditory system development. In C. I. Berlin (Ed.), Hearing science (pp. 109-157). San Diego, CA: CollegeHill Press.

SChneider, B., Trehub, S., \& Bull, D. (1980). High-frequency sensitivity in infants. Science, 207, 1003-1004.

SChNeider, B., Trehub, S., Morrongiello, B., \& Thorpe, L. (1986). Auditory sensitivity in preschool children. Journal of the Acoustical Society of America, 79, 447-452.

Stapells, D., Picton, T., \& SmIth, A. (1982). Normal hearing threshold for clicks. Joumal of the Acoustical Society of America, 72, 74-79.

Teas, D., Klein, A., \& Kamer, S. (1982). An analysis of auditory brainstem responses in infants. Hearing Research, 7, $19-54$.

Trehub. S., Schneider, B., \& Endman, M. (1980). Developmental changes in infants' sensitivity to octave-band noises. Journal of Experimental Child Psychology, 29, 282-293.

Trehub, S., Schneider, B., Morrongiello, B. \& Thorpe, L. (1988). Auditory sensitivity in school-age children. Jourmal of Experimental Child Psychology, 46, 273-285.

Werner, L., \& Feeney, M. P. (1990, April). Pure-tone sensitivity of 2- to 4-week-old infants assessed with auditory reinforcement. Paper presented at the International Conference for Infant Studies, Montreal.

Werner, L., \& Gillenwater, J. (1990). Pure-tone sensitivity of 2to 5-week-old infants. Infant Behavior \& Development, 13, 355-375. Worthington, D.. \& Peters, J. (1980). Quantifiable hearing and no ABR: Paradox or error? Ear \& Hearing, 1, 281-285.

YOST, W., \& KLEIN, A. (1979). Thresholds of filtered transients. Audiology, 18, 17-23.

Zerlin, S. , Naunton, R. (1975). Physical and auditory specifications of third-octave clicks. Audiology, 14, 135-143.

\section{NOTES}

1. Data for infant and adult subjects tested with $4-\mathrm{kHz}$ stimuli have been previously reported in Berg (1991).

2. One consequence of infants' exceptionally high thresholds for brief stimuli is that starting level of the adaptive run, set $20 \mathrm{~dB}$ above adult threshold, was generally above infant threshold for noise bursts, but 
below threshold for clicks. To assess the effect of this procedural difference, infants' threshold runs were divided at the median according to the difference between starting level and estimated threshold. The two resulting groups were compared on several measures, including total number of trials required to estimate threshold, percentage of hits and false alarms after the first reversal, and the range of signal levels contributing to the threshold estimate. Analysis of variance indicated that none of these measures was significantly different for threshold runs above and below the median. Inspection of individual subjects' protocols suggested that the fairly large initial step size $(8 \mathrm{~dB})$ allowed intensity levels to converge rapidly on threshold regardless of starting level.

3. Computer simulations were carried out to determine how variations in observed false-alarm rate and distraction would be expected to alter threshold estimates obtained with the current procedure. As used here, "distraction" refers to a state of task-irrelevant attention, typically directed toward objects displayed at midline by the entertainer and reflected in the incidence of missed probe trials. It was assumed that the probability of a head turn on distraction trials is zero. One thousand simulated threshold runs were averaged for each parameter value. The probability of a head turn on a given trial was computed as

$$
p(\mathrm{HT})=[p(\mathrm{c})+(1-p(\mathrm{c}))(p(\mathrm{fa}))][1-p(\mathrm{~d})],
$$

where $p(\mathrm{fa})$ is the probability of a head turn on a nonsignal trial, $p(\mathrm{~d})$ is the probability of distraction, and $p(c)$ is the logistic function

$$
p(c)=1 /(1+\exp (-L / S)),
$$

where the parameters $L$ and $S$ refer to stimulus level and spread of the psychometric function, respectively.

In general, false alarms had little effect on thresholds at low rates, but estimated threshold decreased rapidly as probability of false alarms approached and exceeded .5. As false-alarm rate increased from 0 to the maximum acceptable rate of .33 , estimated threshold decreased by less than $2 \mathrm{~dB}$. For the mean false-alarm rate of .18 obtained for infants in the present study, the decrease in threshold was approximately $1 \mathrm{~dB}$. Changes in threshold due to distraction were considerably larger; an increase in probability of distraction from 0 to .2 increased the estimated threshold by 5-7 dB when false-alarm rates were within acceptable limits. Varying the spread of the psychometric function from 1.5 to 3.0, intended to cover the range from typical adult functions to the more shallow functions reported for young infants (see, e.g., Olsho et al., 1988), produced only minimal effects.

(Manuscript received November 22, 1991; revision accepted for publication February 9, 1993.) 